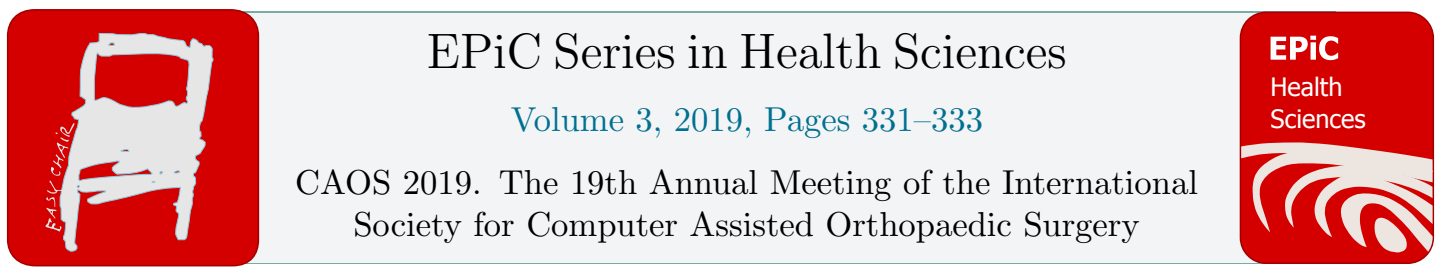

\title{
What factors influence surgeon cervical posture and perceived workload during TKA?
}

\author{
Laura Y. Scholl ${ }^{1}$, Frank Kolisek ${ }^{2}$, Antonia F. Chen, Anil Bhave, Adam \\ Freedhand, Emily Hampp ${ }^{1}$, Vincent Alipit ${ }^{1}$, Michael A. Mont ${ }^{2}$, Anil Bhave ${ }^{3}$, \\ and Antonia F. Chen ${ }^{4}$ \\ ${ }^{1}$ Stryker, Mahwah, NJ, USA \\ ${ }^{2}$ Lenox Hill Hospital, New York, NY, USA \\ ${ }^{3}$ Sinai Hospital Rubin Institute, Baltimore, MD, USA \\ ${ }^{4}$ Birgham and Woman's Hospital, Boston, MA, USA \\ laura.scholl@stryker.com, emily.hampp@stryker.com, vincent.alipit@stryker.com, \\ mmont@northwell.edu, anilbhave@yahoo.com, antoniachen1@gmail.com
}

\begin{abstract}
Orthopaedic surgery is a mentally and physically demanding procedure for surgeons. Studies reported $44-66 \%$ of surgeons surveyed have had a work-related injury attributed to poor surgeon posture. The purpose of this study was to understand how surgical variables may affect a surgeon's posture and workload when performing TKA. Variables included: influence of level of surgical experience, type of surgical procedure, and specific surgical tasks.

Two experienced surgeons, with a median 22-years surgical experience, and 2 surgeons, currently in their fellowship training, each performed 3 manual TKAs (MTKA) and 3 robotic assisted TKAs (RATKA) using a cadaveric setup. Kinematic sensors were placed on the occiput and T3 to measure flexion of the head and neck. Surgeons were surveyed to assess their physical and mental effort using a 1-10 scale (1 being least effort).

Compared to the fellows, experienced surgeons had reduced occiput and T3 angles for MTKA (28.0 vs. $38.7^{\circ}, 4.2$ vs. $\left.15.7^{\circ}\right)$ and RATKA (18.0 vs. $29.2^{\circ}, 4.8$ vs. $\left.13.2^{\circ}\right)$ as well as reduced mental and physical effort. Considering surgical procedure, all surgeons had reduced occiput angles for RATKA compared to MTKA. Considering surgical task, surgical application (MTKA vs. RATKA) had greater influence on cervical angles for the fellows group.

All three factors influenced the surgeon's posture and workload. Occiput angle was reduced by approximately $10^{\circ}$ during RATKA, which is attributed to the surgeon standing in a more upright position, to visualize the robotic screen during cutting and trialing. Robotics may help newer surgeons better visualize knee balancing during TKA, easing the process of balancing and trialing.
\end{abstract}


What factors influence surgeon cervical posture and perceived workload during TKA? L. Scholl et al.

\section{Introduction}

Orthopaedic surgery is a mentally and physically demanding procedure for surgeons. Studies have reported 44-66\% of surgeons surveyed have had a work-related injury, which was attributed to poor surgeon posture.1-2 Literature indicates multiple factors that can influence a surgeon's incidence of injury.3-4 The aim of this study was to understand how three specific variables may affect a surgeon's posture and workload, including: (1) surgeon level of experience, (2) surgical procedure (manual (MTKA) vs. robotic assisted TKA (RATKA)), and (3) specific surgical tasks.

\section{Materials and Methods}

24 TKAs were performed on 12 cadavers by 2 high-volume surgeons (median 22 years surgical experience) and 2 surgeons currently in fellowship training. Each surgeon performed 3 MTKAs and 3 RATKAs. Ergonomic data was collected with kinematic sensors placed on the occiput and T3 to measure flexion and extension of the head and neck relative to a baseline. Workload was assessed with a series of questions after each surgery to assess physical and mental effort, providing scores 1 to 10 , where a higher score correlated to increased effort. Cervical posture and workload questionnaire data were used to compare influence of surgical and surgeon related variables. Surgical tasks common between MTKA and RATKA included, femoral bone cutting, tibial bone cutting, and trialing.

\section{Results}

Summary of cervical posture and workload efforts are provided in Figures $1 \& 2$, respectively. Level of Experience: Considering average occiput and T3 angles, experienced surgeons had reduced angles compared to the fellows for MTKA and RATKA. Considering overall mental and physical effort, experienced surgeons rated TKA less demanding than the fellows for MTKA and RATKA. Surgical Procedure: Experienced surgeons and fellows had reduced occiput angles for RATKA compared to MTKA. Fellows had reduced T3 angle for RATKA compared to MTKA, whereas, experienced surgeons had a similar angle for MTKA and RATKA. Surgical Task: Considering cervical posture by task, fellows had larger occiput and T3 angles when compared to experienced surgeons. When broken down by task, it was noticed that the type of surgical application (MTKA vs. RATKA) had a greater influence on cervical angles when considering the fellow compared to the experienced group. From the questionnaires, the task with the greatest difference in mental effort between MTKA and RATKA for the experienced group was femoral bone cutting. For the fellows group, the largest difference in mental demand was noticed with trialing.

\section{Discussion and Conclusion}

Surgeon level of experience, surgical procedure, and surgical task all influenced the surgeon's posture and workload. Mean occiput angle for both surgeon experience groups was reduced by approximately $10^{\circ}$ during RATKA. This reduction may be attributed to the surgeon standing in a more upright position, to visualize the robotic screen during cutting and trialing, which reduces the time a surgeon may bend their neck to look into the knee joint for the same tasks in MTKA. Reduced mental effort for fellows when performing RATKA trialing should be further explored. Robotics may help newer surgeons better visualize knee balancing during TKA, easing the process of balancing and 
What factors influence surgeon cervical posture and perceived workload during TKA? L. Scholl et al.

resulting in a more straight-forward knee during trialing, which may be less evident in more experienced surgeons. These findings provide initial insights into which factors may have a larger influence on a surgeon's posture and workload. Additional studies in a clinical setting are needed to fully understand these relationships.

\section{References}

[1] Alqahtani et al. JoA 31(2016)1194-1198.

[2] Davis et al. J Bone Joint Surg Am. 2013;95:e107(1-6)

[3] Abdollahzade et al. Health Promotion Perspectives 2016,6(1) 17-22. 